\title{
Intra- and interhemispheric integration of tactual and visual spatial information
}

\author{
RUTH JONES and DIGBY ELLIOTT \\ McMaster University, Hamilton, Ontario, Canada
}

\begin{abstract}
The subjects performed a tactuospatial-visuospatial matching task in which stimuli were presented either to the same sensory field or to opposite fields. Although there was an overall left-side/right-hemisphere advantage for this task, intrahemispheric matching was superior to interhemispheric matching. The results of the study provide evidence against a strong model of unilateral specialization for spatial processing.
\end{abstract}

In the last two decades, a number of studies have provided evidence of right-hemisphere superiority in visuospatial (Berlucchi, Brizzolara, Marzi, Rizzolatti, \& Umiltá, 1979; Kimura, 1969; Kimura \& Durnford, 1974) and tactuospatial (Benton, Varney, \& Hamsher, 1978; Carnahan \& Elliott, 1987; Roy \& MacKenzie, 1978; Witelson, 1974) perception. As with hemispheric specialization for speech and language tasks, however, there is little agreement as to the interpretation of these lateral advantages. Right-hemisphere superiority for a particular spatial task may be due to the fact that only the right hemisphere can perform the task in question, thus requiring the interhemispheric transfer of spatial information when that information is presented to the left hemisphere. Alternately, right-hemisphere superiority may be due to the relative efficiency of that hemisphere in processing spatial information (see Moscovitch, 1986, for discussion). Although it is difficult to test these two positions using traditional sensory field techniques, experimental paradigms that require the interhemispheric integration of information hold some promise (e.g., Umiltá, Rizzolatti, Anzola, Luppino, \& Porro, 1985).

In this experiment, we had subjects match shapes (Witelson, 1974) presented to the right or the left hand to visual representations of the shapes presented to the right or the left visual field. With this sort of spatial task, one would predict an overall left-side/right-hemisphere advantage. Of greater interest was how subjects would perform when one or both stimuli were presented to the left hemisphere. A strong unilateral specialization position would predict performance to be related to the amount of involvement of the specialized hemisphere. Specifically, the two interhemisphere conditions (left field/right hand, right field/left hand) should be superior to the con-

This research was supported by grants from the Natural Sciences and Engineering Research Council of Canada and the Ontario Mental Health Foundation. We thank Gina Loverro and Janie Palmer for preparing the manuscript and Tim Lee for helpful comments. Address correspondence to Digby Elliott, Motor Behaviour Laboratory, School of Physical Education and Athletics, McMaster University, 1280 Main St. West, Hamilton, ON L8S 4K1, Canada. dition in which tactual and visual information were both presented to the left hemisphere (right field/right hand), since this latter situation would require the most interhemispheric transfer of information. However, models advocating bilateral representation with superiority of one or the other hemisphere for certain functions (i.e., a relative efficiency position) may predict intrahemispheric integration of information to be more efficient/faster than interhemispheric crosstalk.

\section{METHOD}

\section{Subjects}

The subjects were 12 male undergraduate students who received course credit for their participation. All subjects were right-handed as determined by Bryden's (1977) modified version of the Oldfield handedness questionnaire, and all had normal or corrected-to-normal vision.

\section{Materials and Equipment}

The tactual stimuli were 10 nonsense shapes designed to be meaningless in order to discourage verbal labeling (Witelson, 1974). The shapes were cut from rubber and measured approximately $4 \times 4 \times .5 \mathrm{~cm}$. They were glued to $10 \times 10 \mathrm{~cm}$ pieces of cardboard for presentation.

A barrier with two openings for the hands was positioned directly in front of the seated subject. The barrier prevented the subject from seeing the tactual stimuli.

The visual stimuli were the visual analogues of the 10 nonsense shapes. They were presented on a $28 \times 43 \mathrm{~cm}$ screen with a slide projector equipped with tachistoscopic shutter (100-msec exposure duration). The stimuli were presented $3.2^{\circ}$ either to the right or left of a fixation dot that was visible in the preexposure field (viewing distance $180 \mathrm{~cm}$ ). The shapes subtended an angle of approximately $1.4^{\circ}$.

\section{Procedure}

At the beginning of a trial, the subject was instructed to place either the right or the left hand through the appropriate hole in the barrier. The subject was required to have his fingers raised and his wrist in contact with the table top. A tactual stimulus was then slid into place under the subject's hand, and the subject was permitted to feel the stimulus with his fingertips for a period of $5 \mathrm{sec}$. After the 5-sec palpitation period, the subject removed his hand and was instructed to fixate the dot on the screen. A "ready" signal was given, and 1-2 sec later a visual target stimulus was presented. The subject's task was to judge whether the visual stimulus was the same as or different from the tactual stimulus on that trial. The subject's vocal response stopped a digital timer.

There were several practice trials followed by 80 experimental trials. Half the trials were same trials, with each of the 10 stimuli presented to each of the four hand/visual-field combinations. For different trials, visual distractors were randomly selected from the remaining pool of 
9 shapes. Thus the design involved a 2 (hand) $\times 2$ (visual field) $\times 2$ (same-different) within-subject factorial arrangement.

Three separate randomizations of trials were used, with 4 subjects participating in each.

\section{RESULTS AND DISCUSSION}

The dependent variables in this study were number of errors and mean voice reaction time. These measures were analyzed in separate 2 (type of trial: same, different) $\times$ 2 (hand) $\times 2$ (visual field) $\times 12$ (subject) repeatedmeasures analyses of variance.

The error analysis revealed only a main effect for type of trial $[F(1,11)=9.9, p<.01]$, indicating that subjects made more errors on same trials (1.71) than on different trials (.52). None of the other main effects or interactions in the analysis approached conventional levels of significance $(p>.15)$.

The voice reaction time analysis revealed interactions involving hand and visual field $[F(1,11)=14.2, p<.01]$ and type of trial and visual field $[F(1,11)=10.7$, $p<.01]$. As is evident in Figure 1, subjects performed most quickly when both tactual and visual information were presented to the left side/right hemisphere. Post hoc analysis of the interaction (Tukey $a, p=.05$ ) revealed that although the left-hand/left-field condition was superior to the two interhemispheric conditions, it did not differ from the right-hand/right-field condition. This intrahemispheric superiority finding provides evidence against strong models of unilateral specialization (see Allen, 1983, for a review) and indicates that the left hemisphere is able to perform some spatial processing. Thus righthemisphere advantages for the perception of spatial arrangement appear to be due to the relative efficiency of

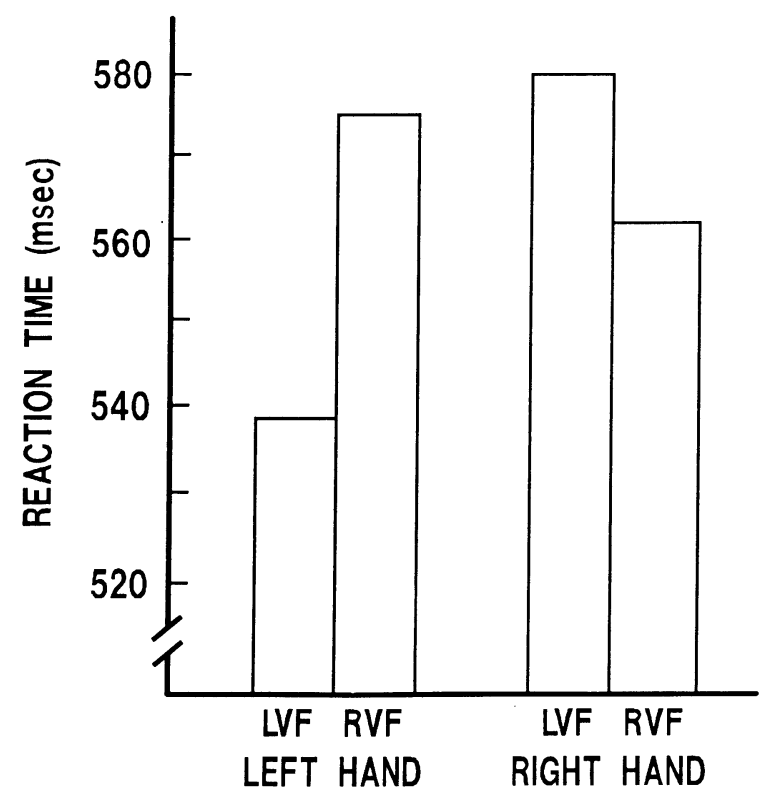

Figure 1. Mean reaction time (in msec) as a function of visual field and hand.

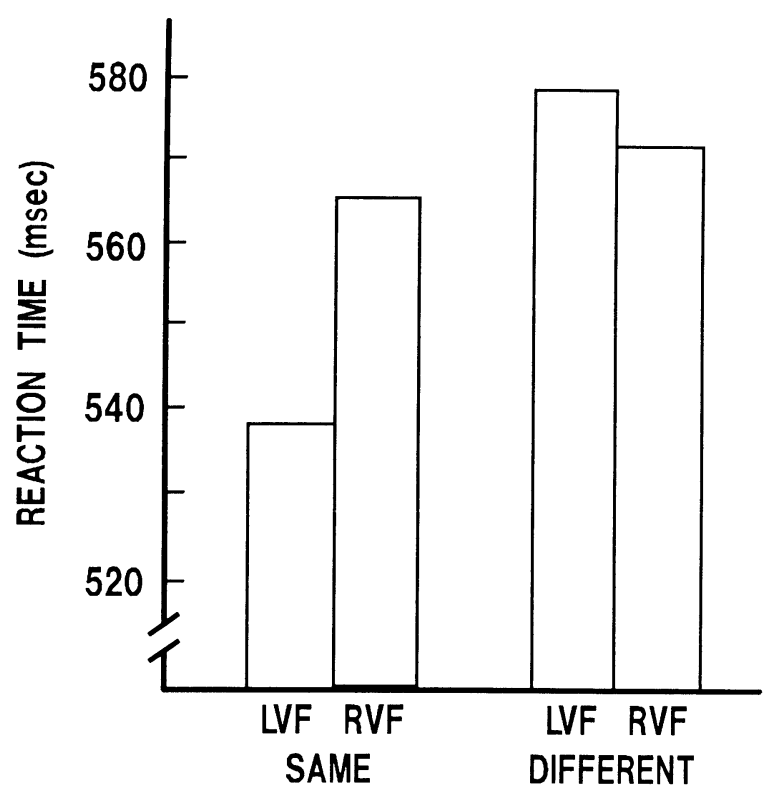

Figure 2. Mean reaction time (in msec) as a function of visual field and type of trial.

that hemisphere in processing spatial information (Moscovitch, 1986).

As depicted in Figure 2, the type of trials $\times$ visual field interaction indicates that although there was a left visual field advantage for same trials, reaction times on different trials were uninfluenced by field (Tukey $a, p=.05$ ). This left visual field advantage for "same" judgments is the reverse of a finding reported by Egeth and Epstein (1972) for decisions about visual letter pairs. Since in our experiment we tried to create stimuli to which subjects were unable to attach verbal labels, perhaps " $s a m e$ " judgments in this experiment were based on a more holistic, Gestalt type of processing than is strategically viable in a letter-matching situation. Although this would explain the left visual field advantage for same trials, why this phenomenon does not hold for different trials is unclear. Perhaps, as Semmes (1968) and Cohen (1972) suggested, judgments of similarity and dissimilarity require different types of processing that may depend more or less on mechanisms associated with the right and left cerebral hemispheres.

In summary, then, the results of this experiment indicate that intrahemispheric matching of tactual and visual spatial stimuli is more efficient than interhemispheric matching. Although there appears to be a processing advantage when both stimuli are presented to the right hemisphere, both hemispheres have the ability to process spatial information.

\section{REFERENCES}

Allen, M. (1983). Models of hemispheric specialization. Psychological Bulletin, 93, 73-104.

Benton, A. L., VARney, N. R., \& HAMSher, K. DE S. (1978). Lateral 
differences in tactile direction perception. Neuropsychologia, 16, 109-114.

Berlucchi, G., Brizzolara, D., Marzi, C. A., Rizzolatti, G., \& UMILTÁ, C. (1979). The role of stimulus discriminability and verbal codability in hemispheric specialization for visuospatial tasks. Neuropsychologia, 17, 195-202.

BRYDEN, M. P. (1977). Measuring handedness with questionnaires. Neuropsychologia, 15, 617-624.

Carnahan, H., \& Ellotot, D. (1987). Pedal asymmetry in the reproduction of spatial locations, Cortex, 23, 157-159.

CoHEN, G. (1972). Hemispheric differences in a letter classification task. Perception \& Psychophysics, 11, 139-142.

Egeth, H., \& Epstein, J. (1972). Differential specialization of the cerebral hemispheres for the perception of sameness and difference. Perception \& Psychophysics, 12, 218-220.

KimurA, D. (1969). Spatial localization in left and right visual fields. Canadian Journal of Psychology, 23, 445-448.

Kimura, D., \& DuRnFord, M. (1974). Normal studies on the function of the right hemisphere in vision. In S. Dimond \& J. Beaumont (Eds.), Hemisphere function in the human brain (pp. 25-47). New York: Wiley.

Moscovitch, M. (1986). Afferent and efferent models of visual perceptual asymmetries: Theoretical and empirical implications. Neuropsychologia, 24, 91-114.

Roy, E. A., \& MacKenziE, C. L. (1978). Handedness effects in kinesthetic spatial location judgements. Cortex, 14, 250-258.

Semmes, J. (1968). Hemispheric specialisation: A possible cue to mechanism. Neuropsychologia, 6, 11-26.

Umiltá, C., Rizzolatti, G., Anzola, G. P., Luppino, G., \& Porro, C. (1985). Evidence of interhemispheric transmission in laterallity effects. Neuropsychologia, 23, 203-213.

Witelson, S. F. (1974). Hemispheric specialization for linguistic and nonlinguistic tactual perception using a dichotomous stimulation technique. Cortex, 10, 3-17.

(Manuscript received for publication July 23, 1987.) 